\title{
MOLECULAR GAS EVOLUTION ACROSS A SPIRAL ARM IN M51
}

\author{
Fumi Egusa ${ }^{1,3}$, Jin KodA ${ }^{2}$, AND Nick ScOville ${ }^{1}$ \\ ${ }^{1}$ California Institute of Technology, MS 249-17 Pasadena, CA 91125, USA; fegusa@ir.isas.jaxa.jp \\ 2 SUNY Stony Brook, Stony Brook, NY 11794, USA \\ Received 2010 July 7; accepted 2010 November 10; published 2010 December 17
}

\begin{abstract}
We present sensitive and high angular resolution $\mathrm{CO}(1-0)$ data obtained by the Combined Array for Research in Millimeter-wave Astronomy observations toward the nearby grand-design spiral galaxy M51. The angular resolution of 0.77 corresponds to $30 \mathrm{pc}$, which is similar to the typical size of giant molecular clouds (GMCs), and the sensitivity is also high enough to detect typical GMCs. Within the $1^{\prime}$ field of view centered on a spiral arm, a number of GMC-scale structures are detected as clumps. However, only a few clumps are found to be associated with each giant molecular association (GMA) and more than $90 \%$ of the total flux is resolved out in our data. Considering the high sensitivity and resolution of our data, these results indicate that GMAs are not mere confusion with GMCs but plausibly smooth structures. In addition, we have found that the most massive clumps are located downstream of the spiral arm, which suggests that they are at a later stage of molecular cloud evolution across the arm and plausibly are cores of GMAs. By comparing with $\mathrm{H} \alpha$ and $\mathrm{Pa} \alpha$ images, most of these cores are found to have nearby star-forming regions. We thus propose an evolutionary scenario for the interstellar medium, in which smaller molecular clouds collide to form smooth GMAs at spiral arm regions and then star formation is triggered in the GMA cores. Our new CO data have revealed the internal structure of GMAs at GMC scales, finding the most massive substructures on the downstream side of the arm in close association with the brightest $\mathrm{H}$ II regions.
\end{abstract}

Key words: galaxies: individual (M51 or NGC 5194) - galaxies: spiral - ISM: clouds - ISM: molecules

Online-only material: color figures

\section{INTRODUCTION}

As all the stars are formed from the interstellar medium (ISM), the evolution of physical and chemical conditions of ISM is an important clue in understanding star formation, its history, and galaxy formation. Spiral galaxies have often been studied with this goal; they are actively forming stars in spiral arms and the sequence of the ISM evolution from molecular gas to young stars can be investigated across the arms. There have been many discussions on the nature of the spiral structure. Lin \& Shu (1964) described it as a quasi-stationary structure sustained by density waves, while tidal waves due to interactions with a companion galaxy or shear motions in the galactic disk can also create transient spiral structures (e.g., Toomre \& Toomre 1972).

With its proximity, nearly face-on disk and grand-design spiral structure, the Whirlpool galaxy, M51 or NGC 5194, has been a popular target for both observational and theoretical studies. Strong streaming motions found in the two spiral arms (Aalto et al. 1999; Shetty et al. 2007) indicate the existence of density waves in this galaxy, while it has been suggested that the spiral structure is not fully stable across time or radius (e.g., Meidt et al. 2008; Dobbs et al. 2010). An interaction with the companion galaxy, NGC 5195, has been thought to enhance or even trigger the prominent spiral structure in the disk of M51. Molecular gas in this grand-design spiral galaxy traced by dust lanes and $\mathrm{CO}$ emission lines has been found upstream of star-forming regions traced by $\mathrm{H} \alpha$ (e.g., Vogel et al. 1988; Rand \& Kulkarni 1990). Tilanus et al. (1988) found similar spatial displacement between non-thermal and thermal radio continuum; spiral arms seen in the non-thermal component are coincident with dust lanes while most of the thermal component

\footnotetext{
3 Current address: Institute of Space and Astronautical Science, Japan Aerospace Exploration Agency, Sagamihara, Kanagawa 252-5210, Japan.
}

peaks agree with $\mathrm{H}$ II regions. These offsets, also found in other spiral galaxies, are consistent with the density wave theory and represent the timescale for molecular clouds to form stars (e.g., Roberts 1969). Variations of physical properties across the arms thus correspond to the evolution of the ISM in spiral galaxies. Egusa et al. (2009) utilized offsets between $\mathrm{CO}$ and $\mathrm{H} \alpha$ to derive a star formation timescale and pattern speed in nearby spiral galaxies.

Given that the typical width of spiral arms is about $500 \mathrm{pc}$, investigating the ISM evolution across the arms requires images with a high spatial resolution, which has been difficult especially for radio observations. Instead, comparison between arm and interarm regions has been used to diagnose how the spiral structure affects the ISM properties. From the ${ }^{12} \mathrm{CO}(1-0)$ data covering the entire disk of M51 taken with the Combined Array for Research in Millimeter-wave Astronomy (CARMA) and Nobeyama $45 \mathrm{~m}$ telescope, Koda et al. (2009, hereafter K09) found that giant molecular associations (GMAs; $\gtrsim 10^{7} M_{\odot}$; Vogel et al. 1988) are only in arm regions while giant molecular clouds (GMCs; $10^{5}-10^{6} M_{\odot}$; Solomon et al. 1979a) are in both arm and interarm regions. On the other hand, bright young stars delineate narrow spiral arms, while older stars are distributed more smoothly over a galactic disk (e.g., Sandage 1961). For M51, Scoville et al. (2001) cataloged H II regions from a highresolution $\mathrm{H} \alpha$ image taken with the Hubble Space Telescope $(H S T)$ and found that about half of them are confined to the arm region which occupies only quarter of the disk area. It is thus expected that GMAs play a critical role in massive star formation in the spiral arms.

However, the typical size of GMAs and $\mathrm{H}$ II regions are quite different $-\gtrsim 200 \mathrm{pc}$ and $\sim 30 \mathrm{pc}$, respectively. Thus, the key to understanding the initiation of massive OB star clusters is the internal structure of GMAs. K09 measured the surface density of GMAs to be similar to that of typical GMCs and 
suggested that GMAs are not simply overlapping of GMCs but are distinct structures where the entire volume is virtually filled with molecular gas. Nevertheless, the spatial resolution of their data $(\sim 200 \mathrm{pc})$ is not high enough to resolve GMAs. Since previous $\mathrm{CO}$ observations with high spatial resolution similar to the size of GMCs and H II regions have only been done for the central region of galaxies or for dwarf and flocculent spiral galaxies in the Local Group, the internal structure of GMAs in grand-design spiral galaxies has not been investigated before.

To resolve GMAs and detect GMC-scale structures ( $\sim 40 \mathrm{pc})$ in spiral arms, we have carried out new CARMA observations in the CO lines toward M51 with a very high angular resolution $(\sim 0$.7). In this paper, we present results from our new $\mathrm{CO}$ observations and discuss the internal structure of GMAs and the relationship of the brightest substructures to the spiral structure and star-forming regions. At the adopted distance of M51 (8.4 Mpc; Feldmeier et al. 1997), $1^{\prime \prime}$ corresponds to 41 pc.

\section{OBSERVATIONS AND DATA REDUCTION}

The $\mathrm{CO}(1-0)$ line observations were carried out at CARMA in 2008 November through December. This array has six $10 \mathrm{~m}$ dishes and nine $6 \mathrm{~m}$ dishes and thus provides 105 baselines. The full width at half maximum (FWHM) of the primary beam is about $60^{\prime \prime}$ and $100^{\prime \prime}$ for the $10 \mathrm{~m}$ and $6 \mathrm{~m}$ dishes, respectively. The B-array configuration, whose typical angular resolution is $0^{\prime \prime} .8$ at $3 \mathrm{~mm}$, was selected to resolve GMAs and detect GMC-scale structures in M51. A single pointing was centered on the brightest GMA in the K09 data located about 30" from the galactic center in the south-west direction. The pointing center coordinate is (R.A., decl.) $=(13: 29: 50.16,+47: 11: 28.39)$ in $\mathrm{J} 2000$. We used the hybrid correlator mode (three $62 \mathrm{MHz}$ bands for the source and three $500 \mathrm{MHz}$ bands for the calibrator) in order to achieve high spectral resolution for the source and high sensitivity for the calibrator. Channel width for the source is $0.977 \mathrm{MHz}$ or $2.54 \mathrm{~km} \mathrm{~s}^{-1}$ at the frequency of $115 \mathrm{GHz}$. The intermediate frequency (IF) was set to include the ${ }^{12} \mathrm{CO}(1-0)$ line in the upper sideband (USB) and ${ }^{13} \mathrm{CO}(1-0)$ in the lower sideband (LSB). Data were taken through the standard cal-source cycle with 5 minutes integration on the phase calibrator, $1153+495$, which is 16 deg away from the source, and 15 minutes on source. The passband observations were done at the beginning of each track with a brighter source and the data were used to calibrate phase and amplitude offsets between narrow and wide bands as well as the normal passband calibration. The total observing time is about $34 \mathrm{hr}$ with five tracks. In terms of the weather condition, the phase rms was $\sim 150-200 \mu \mathrm{m}$ and the opacity at $230 \mathrm{GHz}$ was $\sim 0.3$, which is typical for $3 \mathrm{~mm}$ observations with the B-array configuration.

Data reduction was done with the Multichannel Image Reconstruction, Image Analysis and Display software (MIRIAD; Sault et al. 1995). The flux of the phase calibrator was derived to be $0.81 \mathrm{Jy}$ at $114.75 \mathrm{GHz}$ (USB) and $0.74 \mathrm{Jy}$ at $110.02 \mathrm{GHz}$ (LSB) from the comparison with bright quasars whose flux had been derived from other observations with planets. The uncertainty in the flux calibration is estimated to be $15 \%(\sim 0.1 \mathrm{Jy})$. The synthesized beam from the natural weighting is $0.73 \times 00^{\prime} .60$ for USB and $0.77 \times 0.62$ for LSB, corresponding to $\sim 31 \mathrm{pc} \times$ $25 \mathrm{pc}$ at the distance of M51. The noise rms $(\sigma)$ measured at central $40^{\prime \prime} \times 40^{\prime \prime}$ in emission-free channels of the dirty map is $12 \mathrm{mJy}^{\text {beam }}{ }^{-1}$ or $2.5 \mathrm{~K}$ for USB and $7.2 \mathrm{mJy} \mathrm{beam}^{-1}$ or $1.5 \mathrm{~K}$ for LSB, with the channel width of $5.08 \mathrm{~km} \mathrm{~s}^{-1}$ as Hanning smoothing was applied. The baseline length ranges 23.9 $\mathrm{k} \lambda$ to $362 \mathrm{k} \lambda$, which corresponds to $8^{\prime \prime} .6$ to 0.60 in angular res- olution or $360 \mathrm{pc}$ to $24 \mathrm{pc}$. Given that the typical width of the molecular spiral arm seen in the K09 CO data is $\sim 10^{\prime \prime}$ or 400 pc, most of the structures smaller than the arm down to $24 \mathrm{pc}$ should be detected.

The dirty map was CLEANed by the use of the MIRIAD task mossdi with the flux cutoff of $1 \sigma$. In order to shorten the time needed to CLEAN, we applied a mask made by the combination of (1) the field of view (FOV) and (2) a mask from the K09 cube data. First, we created the sensitivity map from the primary beam patterns by the task mossen and defined the FOV as where the sensitivity is lower than 1.5 times the one at the pointing center. The diameter of this FOV is 53".25 and pixels outside have been masked out to avoid confusion with elevated noise. Meanwhile, we made the R.A.-decl., R.A.-VEL, and decl.-VEL projections of the K09 cube and manually drew a polygon around emissions to make the mask for the entire galaxy. Within the FOV, only the region inside the K09 mask has been used for CLEANing. We refer to this CLEANed data set as B-array data hereafter.

The integrated intensity or moment $0\left(I_{\mathrm{CO}}=\int T_{\mathrm{CO}} d v\right)$ map was created by integrating the B-array data cube with the velocity range of $385.5-563.2 \mathrm{~km} \mathrm{~s}^{-1}$. The flux cutoff for making the $I_{\mathrm{CO}}$ map was set to be proportional to the local sensitivity: $1 \sigma$ at the pointing center and $1.5 \sigma$ at the edge of the FOV. The resulting $I_{\mathrm{CO}}$ map for the ${ }^{12} \mathrm{CO}(1-0)$ data is shown in the left panel of Figure 1, while the B-array FOV is indicated as a box on the K09 $I_{\mathrm{CO}}$ map in the right panel.

\section{RESULTS AND DISCUSSION}

\subsection{Detecting GMC-scale Structures}

In order to identify each molecular cloud structure, the clumpfind method provided by Williams et al. (1994) has been applied to the CLEANed ${ }^{12} \mathrm{CO}(1-0)$ data cube with the lowest level and contouring step of $1 \sigma$. Both of these two parameters are constant within the FOV. We have then defined detections as clumps (1) within the FOV, (2) with the peak flux whose local signal-to-noise ratio $(\mathrm{S} / \mathrm{N}) \geqslant 4$, and (3) with the FWHM in velocity larger than 0.5 pixel (or $2.54 \mathrm{~km} \mathrm{~s}^{-1}$ ). We have calculated this local $\mathrm{S} / \mathrm{N}$, considering the variable sensitivity within the FOV.

The 34 selected clumps are indicated by circles in the left panel of Figure 1 superimposed on the moment 0 map. The line width and color represents the local $\mathrm{S} / \mathrm{N}$ of each clump; thin blue is for $5>\mathrm{S} / \mathrm{N} \geqslant 4$, while thick red is for $\mathrm{S} / \mathrm{N} \geqslant 5$. The radius measured by the clumpfind is $0.5-1.4$ or $10-60 \mathrm{pc}$, corresponding to the radius of the circles in the figure. The FWHM in R.A. and decl. also fall in the same range with the radius and about one-third of the selected clumps are resolved. While most of the clumps are found in the arm, larger and more massive clumps are located only on the west side of the spiral arm. Assuming a trailing arm, this corresponds to the downstream side. On the other hand, some of the smaller clumps are found in the upstream interarm region (east side of the FOV). In Section 3.2, we discuss it in more detail by comparison with the K09 data.

From Figure 1, we have noticed that there are several emission peaks not selected as clumps. Most of them are close to the edge of the FOV and thus are presumably elevated noise, while some appear sequentially on the upstream side of the spiral arm. We have examined all the clumps with $\mathrm{S} / \mathrm{N} \geqslant 3$ and found that they are superpositions of several weak- and narrow-line clumps at different velocities. As some of them seem to be associated with $\mathrm{H}$ II regions (see Figure 5), they perhaps are real molecular 

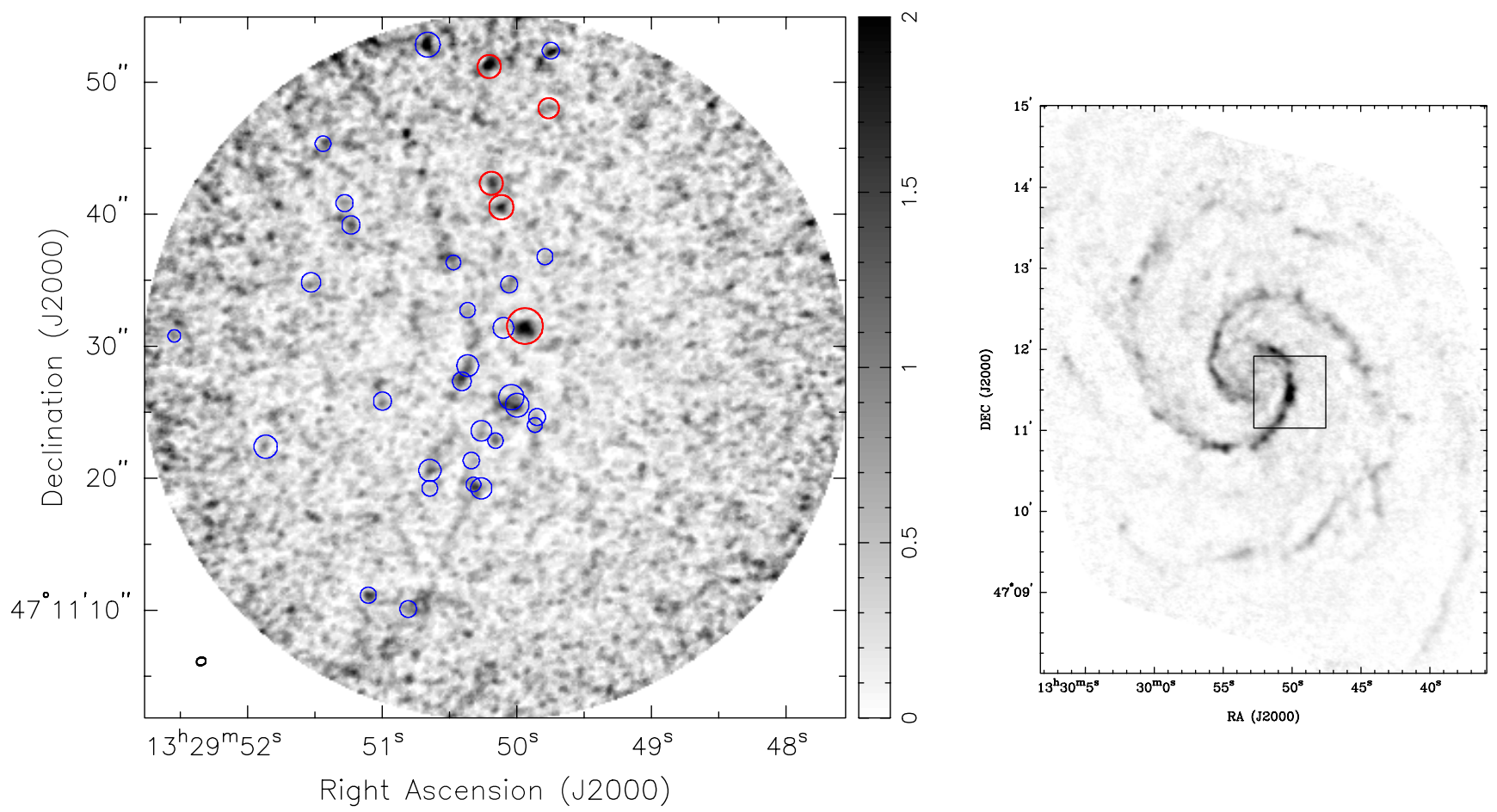

Figure 1. Left: integrated intensity map of ${ }^{12} \mathrm{CO}(1-0)$ data from the B-array CARMA observations. The unit of the gray scale is Jy beam ${ }^{-1} \mathrm{~km} \mathrm{~s}^{-1}$. The synthesized beam $\left(0{ }^{\prime \prime} \cdot 73 \times 00^{\prime} \cdot 60\right)$ is shown as the ellipse on the bottom left corner. Circles superimposed on the gray-scale image indicate clumps detected by clumpfind, with the line width and color corresponding to the local $\mathrm{S} / \mathrm{N}$ of the peak flux; thin blue is for $\mathrm{S} / \mathrm{N} \geqslant 4$ and thick red is for $\mathrm{S} / \mathrm{N} \geqslant 5$. See Section 3.1 for detail. Right: the field of view of the left image is shown as the box overlaid on the lower resolution ${ }^{12} \mathrm{CO}(1-0)$ integrated intensity map from K09. Note that the color scale for the two images is not the same.

(A color version of this figure is available in the online journal.)

clouds, but we need a higher sensitivity to confirm that and do not include them in further discussion.

For the ${ }^{13} \mathrm{CO}(1-0)$ line in the LSB data, the same procedures as the ${ }^{12} \mathrm{CO}(1-0)$ data have been applied and three clumps with $\mathrm{S} / \mathrm{N} \geqslant 4$ have been detected. None of them, however, are found to be associated with the ${ }^{12} \mathrm{CO}(1-0)$ clumps. Lowering the $\mathrm{S} /$ $\mathrm{N}$ cutoff to 3.0, we have detected 162 clumps and found two clumps with $\mathrm{S} / \mathrm{N} \simeq 3.6$ located near ${ }^{12} \mathrm{CO}(1-0)$ clumps; one is located close to the pointing center and coincides with the largest and most massive ${ }^{12} \mathrm{CO}(1-0)$ clump (marked as "d" in Figure 2), while the other is located close to the two ${ }^{12} \mathrm{CO}(1-0)$ clumps $\sim 10^{\prime \prime}$ north of the pointing center ("b" and "c" in Figure 2). The ratio of the peak temperatures of the corresponding clumps is $T_{\text {peak }}\left({ }^{13} \mathrm{CO}\right) / T_{\text {peak }}\left({ }^{12} \mathrm{CO}\right) \sim 0.4$, while the ratio of the total flux is $F_{\text {tot }}\left({ }^{13} \mathrm{CO}\right) / F_{\text {tot }}\left({ }^{12} \mathrm{CO}\right) \sim 0.2$. The average integrated intensity ratio for the Galaxy is $1 / 5.5$ (Solomon et al. 1979b), implying that these massive clumps detected in M51 are in similar condition to GMCs in the Galaxy. As these ${ }^{13} \mathrm{CO}(1-0)$ structures do not meet the $\mathrm{S} / \mathrm{N}$ criterion for the clump selection, we discuss only the ${ }^{12} \mathrm{CO}(1-0)$ results hereafter.

The conversion factor from the ${ }^{12} \mathrm{CO}(1-0)$ integrated intensity to the $\mathrm{H}_{2}$ column density $\left(N\left(\mathrm{H}_{2}\right)=X_{\mathrm{CO}} I_{\mathrm{CO}}\right)$ has been measured in a number of ways. For the Galaxy, $X_{\mathrm{CO}} \simeq 3 \times 10^{20} \mathrm{~cm}^{-2}$ $\left(\mathrm{K} \mathrm{km} \mathrm{s}^{-1}\right)^{-1}$ was derived by comparing the virial mass and the ${ }^{12} \mathrm{CO}(1-0)$ flux of GMCs (e.g., Solomon et al. 1987; Young \& Scoville 1991). More recently, smaller values $(\sim 1.8 \times$ $\left.10^{20} \mathrm{~cm}^{-2}\left(\mathrm{~K} \mathrm{~km} \mathrm{~s}^{-1}\right)^{-1}\right)$ have been reported by comparison with the $\gamma$-ray or far infrared data (Strong \& Mattox 1996; Dame et al. 2001; Grenier et al. 2005). Heyer et al. (2009) derived GMC masses from the ${ }^{13} \mathrm{CO}(1-0)$ emission lines under the local thermodynamic equilibrium (LTE) assumption and found that the LTE masses are factor of $\sim 5$ smaller than the virial masses. The inconsistencies in the $X_{\mathrm{CO}}$ values are due to the combination of uncertainties and/or invalidities of the models and assumptions introduced and imply difficulties in the $X_{\mathrm{CO}}$ determination.

As for the Galaxy, the derived $X_{\mathrm{CO}}$ values for M51 depend largely on data and methods. While the virial masses of GMAs derived by Rand \& Kulkarni (1990) agree with $X_{\mathrm{CO}}=3 \times$ $10^{20} \mathrm{~cm}^{-2}\left(\mathrm{~K} \mathrm{~km} \mathrm{~s}^{-1}\right)^{-1}$, those derived by Adler et al. (1992) based on a different $\mathrm{CO}$ data set suggest $X_{\mathrm{CO}}=1.2 \times 10^{20} \mathrm{~cm}^{-2}$ $\left(\mathrm{K} \mathrm{km} \mathrm{s}^{-1}\right)^{-1}$. Even smaller values have been suggested (Garcia-Burillo et al. 1993; Rand 1993; Nakai \& Kuno 1995). More recently, Schinnerer et al. (2010) applied the large velocity gradient (LVG) modeling to $2^{\prime \prime}$ resolution multi-transition CO data and derived $X_{\mathrm{CO}}=(1.3-1.9) \times 10^{20} \mathrm{~cm}^{-2}\left(\mathrm{~K} \mathrm{~km} \mathrm{~s}^{-1}\right)^{-1}$. In this paper, we adopt $X_{\mathrm{CO}}=1.5 \times 10^{20} \mathrm{~cm}^{-2}\left(\mathrm{~K} \mathrm{~km} \mathrm{~s}^{-1}\right)^{-1}$. The variations of $X_{\mathrm{CO}}$ within the FOV as a function of galactocentric radius should be small enough to be neglected, as the data only cover $53^{\prime \prime}$ or $2.2 \mathrm{kpc}$.

The noise rms $(1 \sigma)$ in the ${ }^{12} \mathrm{CO}(1-0)$ data thus corresponds to $M_{\mathrm{H}_{2}}=2.5 \times 10^{4} M_{\odot}$ within the synthesized beam $(\sim 30 \mathrm{pc})$ and one velocity channel $\left(5.08 \mathrm{~km} \mathrm{~s}^{-1}\right)$. Though the absolute mass of molecular gas could be changed by a factor of two or more according to adopted $X_{\mathrm{CO}}$ values, the sensitivity in mass is still smaller than typical GMCs in any case. Furthermore, the conclusion of this paper does not depend on the $X_{\mathrm{CO}}$ value, since we only focus on the spatial and kinematical information and discuss relative values of mass from the B-array and K09 data.

\subsection{Relationship to Larger Structures}

In the left panel of Figure 2, contours from the K09 $I_{\mathrm{CO}}$ map are overlaid on the B-array $I_{\mathrm{CO}}$ image. We have applied the 

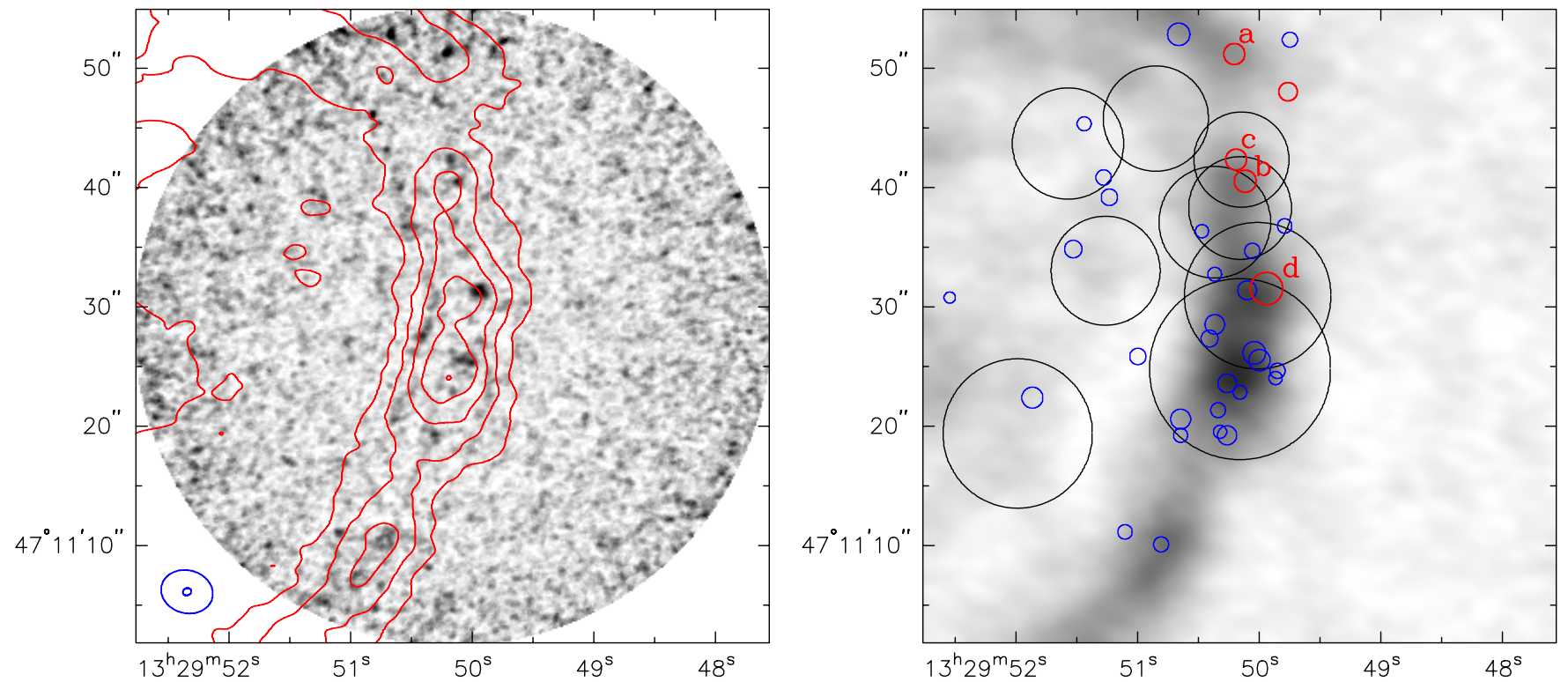

Figure 2. Left: contours from the K09 moment 0 map overlaid on the B-array moment 0 map. The synthesized beams for these two data sets are indicated by ellipses on the bottom left corner. Right: clumps from the B-array data (as circles with blue or red line) and GMAs from the K09 data (as circles with black lines) superimposed on the K09 moment 0 map. See Sections 3.1 and 3.2 for their definition. Clumps whose spectra are shown in Figure 3 are marked with "a"-"d."

(A color version of this figure is available in the online journal.)

clumpfind algorithm to the K09 data cube and defined GMAs as clumps with $M>10^{7} M_{\odot}$. Following the selection criteria for the B-array data, clumps on the data edge have been excluded. These selected GMAs are shown as open black circles in the right panel of Figure 2 together with the detected clumps in the B-array data in the same manner as Figure 1. The background image is the K09 moment 0 map. From this figure, we find that GMAs are not filled with the GMC-scale clumps. As the $1 \sigma$ sensitivity of the B-array data is about 10 times smaller than the typical mass of GMCs, we should have detected tens or more of them if GMAs were confused with GMCs. This result strongly indicates that GMAs are not confusions nor overlappings of GMCs.

The total flux within the FOV of the B-array data has been measured to be $67 \mathrm{Jy} \mathrm{km} \mathrm{s}^{-1}$ and $1.1 \times 10^{3} \mathrm{Jy} \mathrm{km} \mathrm{s}^{-1}$ for the B-array and K09 data, respectively. As the K09 data include the single-dish observations with the Nobeyama $45 \mathrm{~m}$ telescope, it should represent the total ${ }^{12} \mathrm{CO}(1-0)$ flux. The missing flux in the B-array data is thus $94 \%$, suggesting that most of the CO flux in the spiral arm of M51 should come from either extended structures or molecular clouds smaller than GMCs and supporting the aforementioned indication that GMAs are not collections of GMCs.

Another important clue from comparison of the two $I_{\mathrm{CO}}$ maps is that their peak positions do not coincide; massive clumps detected in the B-array data are shifted a few arcseconds toward the downstream side of the global spiral arm seen in the K09 map. To investigate this difference further with the velocity information, we have made spectra of B-array and K09 data at the positions of the selected clumps. The box size for the average spectra was set to be the same as the diameter of each clump derived by clumpfind. In Figure 3, the sample spectra for massive clumps are displayed. The flux difference mentioned above is also clearly seen in the spectra. From these spectra, we have found that massive clumps have offsets in peak velocity between the two data sets. We have defined the velocity offset as

$$
V_{\text {ofs }}=V_{\mathrm{cl}}(\mathrm{B})-V_{\text {peak }}(\mathrm{K} 09),
$$

where $V_{\mathrm{cl}}(\mathrm{B})$ is the peak velocity for clumps measured by the clumpfind and $V_{\text {peak }}(\mathrm{K} 09)$ is the peak velocity in the K09 spectra. The $V_{\text {ofs }}$ is plotted against the mass of each clump in Figure 4. It is clear from this plot that almost all of the clumps with $M_{\mathrm{H}_{2}}>5 \times 10^{5} M_{\odot}$ have negative $V_{\text {ofs }}$. Considering the galactic rotation, negative velocities correspond to the downstream side. Therefore, massive clumps are kinematically as well as spatially located downstream of the spiral arm. This difference indicates that the massive clumps are in a later evolutionary stage compared to the larger scale structures or GMAs. We refer to these massive clumps as GMA cores hereafter.

Smaller clumps on the other hand are located preferentially on upstream of the spiral arm, and little emission is found in the interarm region on downstream. This distribution of small clumps indicates that they are somehow destroyed through the evolution across the spiral arm. Before forming stars, the small clumps $\left(M \sim 10^{5} M_{\odot}\right)$ could collide with each other to form larger clouds such as GMAs. Meanwhile, they would be dissociated into atomic gas or broken up to even smaller clouds $\left(M<10^{5} M_{\odot}\right)$ after forming stars.

\subsection{Relationship to Star-forming Regions}

As already mentioned in Section 1, star-forming regions are located on the downstream side of the spiral arms. Since we have found that GMA cores are also on downstream, it is worth comparing these two components.

The $\mathrm{H} \alpha$ data taken under the HST program (ID: 10452 , PI: S. Beckwith) were obtained from the HST Web site ${ }^{4}$ and cover $\sim 7^{\prime} \times 10^{\prime}$, including the entire galactic disk and the companion galaxy (Mutchler et al. 2005). The Pa $\alpha$ image taken for another HST program (ID: 7237, PI: N. Scoville) was

\footnotetext{
4 http://archive.stsci.edu/prepds/m51/
} 

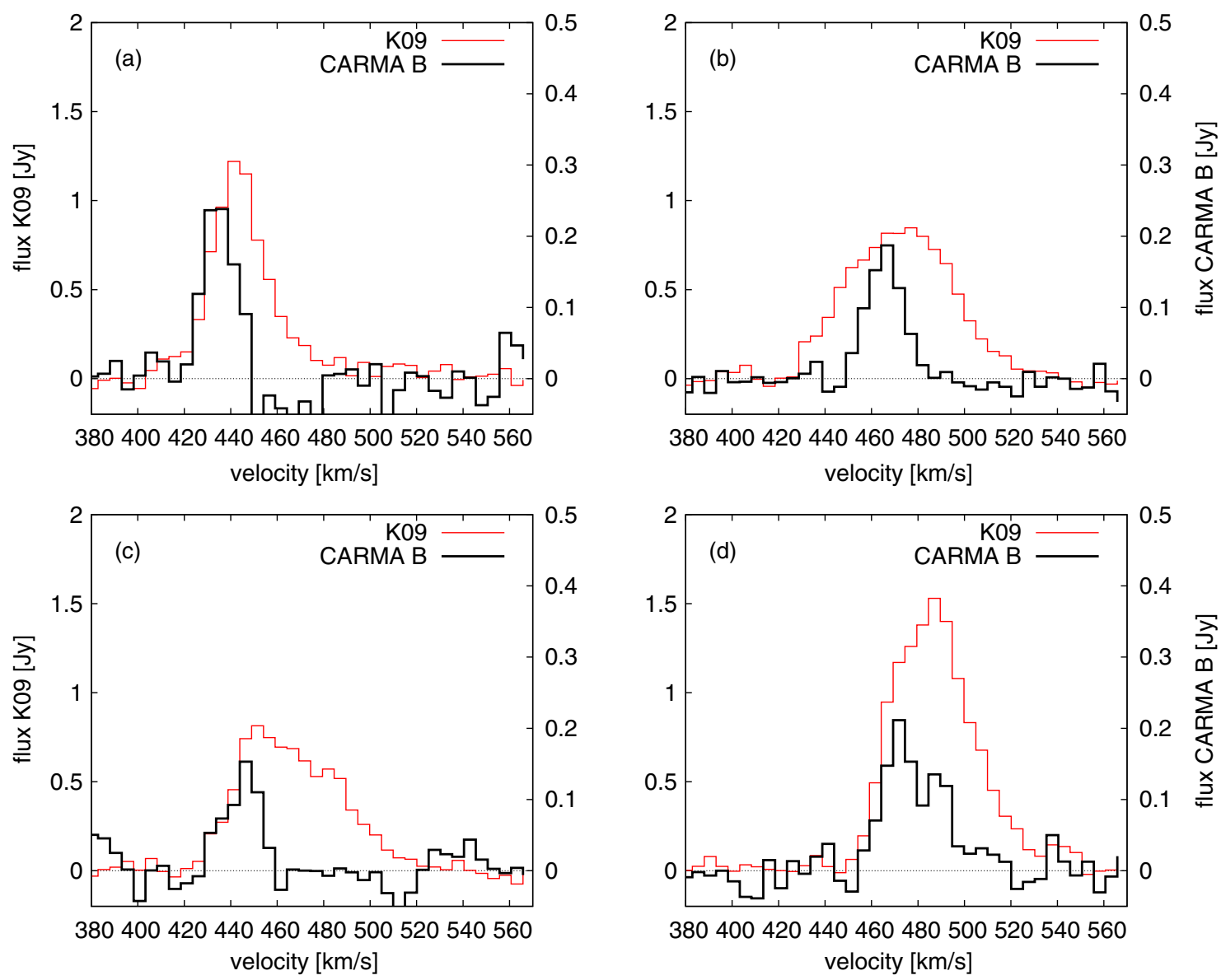

Figure 3. Example of ${ }^{12} \mathrm{CO}(1-0)$ spectra for selected massive clumps, which are indicated in the right panel of Figure 2 . Thin red lines indicate the K09 data while thick black lines indicate the B-array data. Note the difference in flux scale between the two data sets.

(A color version of this figure is available in the online journal.)

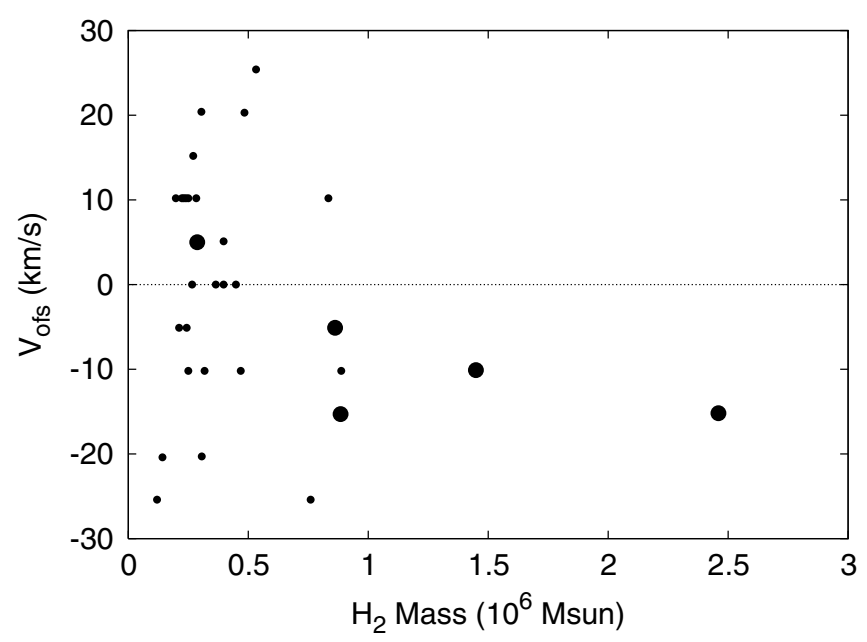

Figure 4. Plot of $V_{\text {ofs }}$ defined in Equation (1) vs. mass of the selected clumps. Larger points represent clumps with $\mathrm{S} / \mathrm{N} \geqslant 5$. A few data points outside the $V_{\text {ofs }}$ range are not shown.

presented by Scoville et al. (2001) and covers the central $3^{\prime}$ of the disk. The astrometry of the $\mathrm{Pa} \alpha$ image was found to be slightly off compared to the $\mathrm{H} \alpha$ image. In order to match their coordinates, we measured the peak position of bright $\mathrm{H}$ iI regions seen in both $\mathrm{H} \alpha$ and $\mathrm{Pa} \alpha$ images and adjusted the coordinates of the $\mathrm{Pa} \alpha$ image through the use of the task ccmap of the Image
Reduction and Analysis Facility (IRAF). ${ }^{5}$ Although the fitting rms from ccmap is as small as 0 '. $^{\prime} 17$ and 0 '. $^{\prime} 13$ for R.A. and decl., respectively, the errors in the coordinates could be larger as $\mathrm{HII}$ regions are extended especially in the $\mathrm{H} \alpha$ image and extinction gradients might affect the flux distribution and thus the peak positions. We estimate the effective uncertainty in the coordinates to be about $40 \mathrm{pc}$ or $1^{\prime \prime}$, which is the typical size of GMCs and $\mathrm{H}$ II regions. In Figure 5, circles indicating the selected clumps are superimposed on the $\mathrm{H} \alpha$ and $\mathrm{Pa} \alpha$ images.

As expected, bright $\mathrm{H}$ II regions are located on the downstream side of the $\mathrm{CO}$ spiral arm seen in the lower resolution K09 data. GMA cores found in the high resolution B-array data, on the other hand, coincide with the $\mathrm{H}$ II regions. This correlation indicates that GMA cores are the site of massive star formation, which is consistent with the result in the previous subsection that they are in a later stage of the molecular gas evolution. Meanwhile, we have found that not all the $\mathrm{H}$ II regions are accompanied by the cores, which implies that GMA cores are short-lived or are not prerequisites for massive star formation.

Given that only the ${ }^{12} \mathrm{CO}(1-0)$ data are available at this resolution, physical conditions such as density and temperature of GMA cores cannot be determined uniquely; high density and high temperature can both produce high $I_{\mathrm{CO}}$. The largest core

\footnotetext{
5 IRAF is distributed by the National Optical Astronomy Observatory, which is operated by the Association of Universities for Research in Astronomy, Inc. under cooperative agreement with the National Science Foundation.
} 

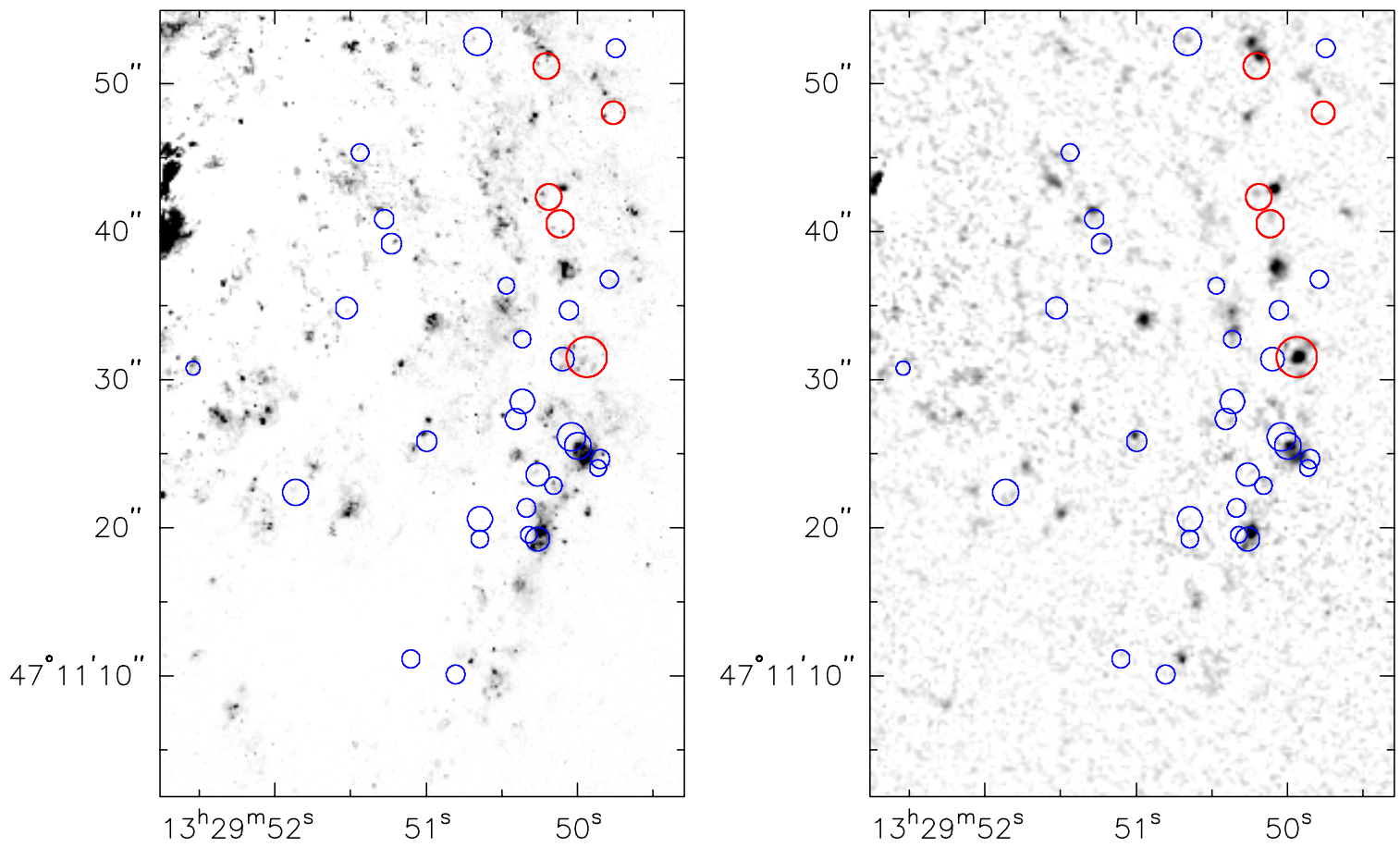

Figure 5. Selected clumps presented as circles (same as Figure 1) on the $H S T \mathrm{H} \alpha$ (left) and Pa $\alpha$ (right) image. One-third from the right edge of the field of view, where no clumps have been detected, is not displayed.

(A color version of this figure is available in the online journal.)

close to the pointing center (marked as "d" in Figure 2) gives a clue to this issue. From Figure 5, we have found that it does not have a counterpart in $\mathrm{H} \alpha$ but in $\mathrm{Pa} \alpha$, indicating high extinction and thus a high column density. From the mass $\left(2.5 \times 10^{6}\right.$ $\left.M_{\odot}\right)$ and radius $(55 \mathrm{pc}$ ) measured by clumpfind, the average $\mathrm{H}_{2}$ column density is derived to be $N\left(\mathrm{H}_{2}\right)=1.6 \times 10^{22} \mathrm{~cm}^{-2}$ for this core. Assuming $A_{V} / N_{\mathrm{H}}=5.3 \times 10^{-22} \mathrm{mag} \mathrm{cm}^{2}$ (Bohlin et al. 1978), the estimated extinction is as high as $A_{V}=17 \mathrm{mag}$. The most plausible detection of ${ }^{13} \mathrm{CO}(1-0)$ at this location is also consistent with the high density. Therefore, at least for this core, we conclude that the high $I_{\mathrm{CO}}$ represents high column density of molecular gas. This agreement in high extinction and density also confirms that the coordinate adjustment for the $\mathrm{Pa} \alpha$ image is correct within the core size or $\sim 1^{\prime \prime}$.

Additionally, it is clear from Figure 5 that the distribution of $\mathrm{H}$ II regions is quite similar in the $\mathrm{H} \alpha$ and $\mathrm{Pa} \alpha$ images, indicating that only a few regions (such as the most massive core mentioned above) are significantly affected by extinction. This means that $\mathrm{H} \alpha$ is a good tracer of the locations of current star formation in most cases and is preferable over extinction-free $24 \mu \mathrm{m}$ for measuring offsets between molecular arm and young stellar arm (Egusa et al. 2009) as it generally provides higher angular resolution.

\subsection{Evolution Scenario Across a Spiral Arm}

The results presented above reveal the structure and distribution of molecular clouds at $30 \mathrm{pc}$ scale for the first time in grand-design spiral galaxies. Based on their properties and relationship to the global $(\mathrm{kpc})$ spiral structure, we here propose a scenario for the ISM evolution across the spiral arm. We should note here that smaller spatial scale across the arm corresponds to shorter timescale, which enables us to describe the scenario in unprecedented detail.
On the upstream side of the spiral arm, molecular clouds of $M_{\mathrm{H}_{2}} \sim 10^{5} M_{\odot}$ collide with each other and probably with smaller clouds to grow into GMAs $\left(M_{\mathrm{H}_{2}} \gtrsim 10^{7} M_{\odot}\right)$. GMAs are presumably distinct and smooth structures and a few GMC-scale cores $\left(\sim 10^{6} M_{\odot}\right)$ are formed within each GMA, which turn out to be the site of OB star formation as they evolve. If not all the gas in cores would turn into stars, the remaining gas in the core should break up to smaller molecular clouds $\left(<10^{5} M_{\odot}\right)$ or become dissociated into atomic hydrogen by strong radiation from young stars. From the star formation law presented by Kennicutt et al. (2007) for M51, the gas consumption timescale measured as the ratio of the total gas mass to the star formation rate is $\sim 10^{9}$ yr. Since the star formation timescale is much shorter $\left(\sim 10^{7} \mathrm{yr}\right.$; Egusa et al. 2009) than this timescale, most of the gas in GMAs is not used by the star formation activity. Such gas remains molecular through the evolutionary sequence, considering that the molecular fraction to the total hydrogen has been estimated as $70 \%-80 \%$ even in the interarm regions (K09).

\section{SUMMARY}

We have carried out high resolution $\mathrm{CO}$ observations toward a spiral arm in the nearby spiral galaxy M51 using the radio interferometer CARMA, in order to (1) resolve GMAs, (2) detect GMC-scale structures, and (3) understand their relationship to the global spiral structure. For the ${ }^{12} \mathrm{CO}(1-0)$ line, the angular resolution and noise rms $(1 \sigma)$ are $0^{\prime \prime} .7 \times 00^{\prime \prime} 6$ (or $\sim 30 \mathrm{pc}$ ) and 12 mJy beam ${ }^{-1}$ (or $2.5 \mathrm{~K}$ ) with the velocity width of $5.1 \mathrm{~km} \mathrm{~s}^{-1}$, respectively. Assuming the $\mathrm{CO}$-to- $\mathrm{H}_{2}$ conversion factor, $4 \sigma$ corresponds to $10^{5} M_{\odot}$ within the beam, which is smaller than the mass of typical GMCs. The spatial resolution is similar to the typical size of GMCs, and thus the data quality is high enough to detect GMC-like structures. 
Within the $1^{\prime}$ FOV, a number of GMC-scale structures referred as clumps are detected in the ${ }^{12} \mathrm{CO}(1-0)$ data. For the ${ }^{13} \mathrm{CO}(1-0)$ line, only two marginal detections have been identified. By comparing with lower resolution ${ }^{12} \mathrm{CO}(1-0)$ data (K09), we have located only a few clumps in each GMA and derived the missing flux to be larger than $90 \%$. These results indicate that GMAs are not mere confusions with GMCs but are plausibly single and smooth structures. Among the detected clumps, we have found that the most massive $\left(\gtrsim 5 \times 10^{5} M_{\odot}\right)$ clumps are located at the downstream side of the spiral arm spatially as well as kinematically. This displacement indicates that they are at a later stage of the ISM evolution across the spiral arm and plausibly are cores of GMAs. Spatial coincidence between the massive clumps and H II regions supports this hypothesis. Smaller clumps on the other hand are found preferentially in the upstream side of the spiral arm.

We thus propose an evolutionary scenario for the ISM in spiral galaxies; as smaller clouds in interarm regions approach spiral arms, they cluster or collide to form GMAs, which are smooth and discrete structures. Within a GMA, a few massive and GMC-scale substructures referred to as GMA cores are formed at a later stage of its lifetime. Star formation is triggered within the GMA cores, and finally the remaining gas in the core is dissociated into atomic gas or broken up to even smaller clouds after stars are formed.

The sensitive and high resolution $\mathrm{CO}$ data from our new CARMA observations have revealed the internal structure of GMAs at GMC scale and its relationship to the spiral arm structure for the first time in external galaxies. This result is an important step to fully understanding the ISM evolution scenario in spiral galaxies.

We are grateful to E. Schinnerer who kindly provided us a draft of her paper prior to publication. Support for CARMA construction was derived from the states of California, Illinois, and Maryland, the James S. McDonnell Foundation, the Gordon and Betty Moore Foundation, the Kenneth T. and Eileen L. Norris Foundation, the University of Chicago, the Associates of the California Institute of Technology, and the National Science Foundation. Ongoing CARMA development and operations are supported by the National Science Foundation under a cooperative agreement and by the CARMA partner universities. This research is partially supported by HST-AR-11261.01. Facility: CARMA

\section{REFERENCES}

Aalto, S., Hüttemeister, S., Scoville, N. Z., \& Thaddeus, P. 1999, ApJ, 522, 165 Adler, D. S., Lo, K. Y., Wright, M. C. H., Rydbeck, G., Plante, R. L., \& Allen, R. J. 1992, ApJ, 392, 497

Bohlin, R. C., Savage, B. D., \& Drake, J. F. 1978, ApJ, 224, 132

Dame, T. M., Hartmann, D., \& Thaddeus, P. 2001, ApJ, 547, 792

Dobbs, C. L., Theis, C., Pringle, J. E., \& Bate, M. R. 2010, MNRAS, 403, 625

Egusa, F., Kohno, K., Sofue, Y., Nakanishi, H., \& Komugi, S. 2009, ApJ, 697, 1870

Feldmeier, J. J., Ciardullo, R., \& Jacoby, G. H. 1997, ApJ, 479, 231

Garcia-Burillo, S., Guelin, M., \& Cernicharo, J. 1993, A\&A, 274, 123

Grenier, I. A., Casandjian, J., \& Terrier, R. 2005, Science, 307, 1292

Heyer, M., Krawczyk, C., Duval, J., \& Jackson, J. M. 2009, ApJ, 699, 1092

Kennicutt, R. C., Jr., et al. 2007, ApJ, 671, 333

Koda, J., et al. 2009, ApJ, 700, L132

Lin, C. C., \& Shu, F. H. 1964, ApJ, 140, 646

Meidt, S. E., Rand, R. J., Merrifield, M. R., Shetty, R., \& Vogel, S. N. 2008, ApJ, 688,224

Mutchler, M., et al. 2005, BAAS, 37, 452

Nakai, N., \& Kuno, N. 1995, PASJ, 47, 761

Rand, R. J. 1993, ApJ, 404, 593

Rand, R. J., \& Kulkarni, S. R. 1990, ApJ, 349, L43

Roberts, W. W. 1969, ApJ, 158, 123

Sandage, A. 1961, The Hubble Atlas of Galaxies (Washington, DC: Carnegie Institution)

Sault, R. J., Teuben, P. J., \& Wright, M. C. H. 1995, in ASP Conf. Ser. 77, Astronomical Data Analysis Software and Systems IV, ed. R. A. Shaw, H. E. Payne, \& J. J. E. Hayes (San Francisco, CA: ASP), 433

Schinnerer, E., Weiß, A., Aalto, S., \& Scoville, N. Z. 2010, ApJ, 719, 1588 Scoville, N. Z., Polletta, M., Ewald, S., Stolovy, S. R., Thompson, R., \& Rieke, M. 2001, AJ, 122, 3017

Shetty, R., Vogel, S. N., Ostriker, E. C., \& Teuben, P. J. 2007, ApJ, 665, 1138

Solomon, P. M., Rivolo, A. R., Barrett, J., \& Yahil, A. 1987, ApJ, 319, 730

Solomon, P. M., Sanders, D. B., \& Scoville, N. Z. 1979a, in IAU Symp. 84, The Large-Scale Characteristics of the Galaxy, ed. W. B. Burton (Dordrecht: Reidel), 35

Solomon, P. M., Sanders, D. B., \& Scoville, N. Z. 1979b, ApJ, 232, L89

Strong, A. W., \& Mattox, J. R. 1996, A\&A, 308, L21

Tilanus, R. P. J., Allen, R. J., van der Hulst, J. M., Crane, P. C., \& Kennicutt, R. C. $1988, \mathrm{ApJ}, 330,667$

Toomre, A., \& Toomre, J. 1972, ApJ, 178, 623

Vogel, S. N., Kulkarni, S. R., \& Scoville, N. Z. 1988, Nature, 334, 402

Williams, J. P., de Geus, E. J., \& Blitz, L. 1994, ApJ, 428, 693

Young, J. S., \& Scoville, N. Z. 1991, ARA\&A, 29, 581 